\title{
Antitrust Analysis for the Internet Upstream Market: A BGP Approach
}

Alessio D'Ignazio and Emanuele Giovannetti

November 2005

CWPE 0554

Not to be quoted without permission 


\title{
Antitrust Analysis for the Internet Upstream Market: a BGP Approach
}

\author{
Alessio D'Ignazio* \\ Emanuele Giovannetti ${ }^{\bullet \bullet}$
}

\begin{abstract}
In this paper we study concentration in the European Internet upstream access market. The possibility of measuring market concentration depends on a correct definition of the market itself; however, this is not always possible, since, as it is the case of the Internet industry, very often Antitrust authorities lack reliable pricing and traffic data. This difficulty motivates our paper. We present an alternative approach based on the inference of the Internet Operators interconnection policies using micro-data sourced from their Border Gateway Protocol tables.

We assess market concentration following a two step process: firstly we propose a price-independent algorithm for defining both the vertical and geographical relevant market boundaries, then we calculate market concentration indexes using two novel metrics. These assess, for each undertaking, both its role in terms of essential network facility and of wholesale market dominance. The results, applied to four leading Internet Exchange Points in London, Amsterdam, Frankfurt and Milan, show that some vertical segments of these markets are highly concentrated, while others are extremely competitive. According to the Merger Guidelines some of the estimated market concentration values would immediately fall within the special attention category.
\end{abstract}

JEL: K21,L40, L86, L96

Keywords: Network Industries, Internet, Market Concentration, Essential Facilities, $B G P$

\footnotetext{
* Faculty of Economics, University of Cambridge.

- Faculty of Economics, University of Cambridge and University of Rome "La Sapienza".

Corresponding author: Emanuele Giovannetti, Senior Research Associate, Faculty of Economics, Austin Robinson Building, Sidgwick Avenue, Cambridge CB3 9DD. E-mail: e.giovannetti@econ.cam.ac.uk.

- We would like to thank Joerge Lepler, Tim Griffin, Randy Bush, John Souter (LINX), Chris Fletcher (LINX), Valeria Rossi (MIX) and the participants at the $7^{\text {th }}$ Euro-IX Forum in Prague September 2005 for their help, useful comments and data. The usual disclaimer applies. The authors would also like to acknowledge EU financing through the $6^{\text {th }}$ Framework Project IST-2004-2012 and from the Isaac Newton Trust, Trinity College, University of Cambridge.
} 


\section{Introduction}

Market power is usually associated to the ability of a firm to raise prices above marginal costs, or above their competitive level, without loss of profits. Competition authorities are interested in market power because of its potential effects in reducing welfare, at least from a static point of view ${ }^{1}$.

In this paper we attempt to measure the extent of market power characterising the Internet upstream access in Europe. The structure of the Internet is indeed highly hierarchical, with a relatively small number of upstream providers (the Internet backbones -IBPs-) that face the interconnection demand arising from a much larger number of downstream operators of smaller dimensions (Internet Service Providers -ISPs-). Our attempt is also related to the ongoing debate on the possibility of introducing some form of regulation in the Internet upstream access market, whose mechanisms are blamed by many countries to be the cause of the persistence of the digital divide ${ }^{2}$.

It is clear that the possibility of correctly measuring market power depends on a correct definition of the market itself; however, this is not always possible, since very often the antitrust authorities lack reliable data. This is indeed what seems to happen for the proposed mergers between Internet backbones. After the early antitrust cases (1998, MCI and WorldCom merger; 2000, MCI-WorldCom and Sprint), new guidelines were introduced in July 2002. Their applicability is however still very problematic for the assessment of the Internet upstream connectivity market, where both interconnection agreements and traffic flows are sealed under confidentiality agreements.

This difficulty motivates our paper. We present an alternative approach to define the relevant market and assess market concentration in the upstream Internet access by using publicly available data. In particular, our analysis is based on the inference of interconnection policies, expressing the real bargaining power of each ISP: these can be essentially grouped into (1) paid transit, a contractual relation characterised by non linear pricing, a typical discrimination practice and (2) peering, a bilateral free interconnection decision based on a reciprocal agreement, again an open form of discrimination formally expressed in the peering policy of every provider. The inference is obtained from the Border Gateway Protocol (BGP) output data (the BGP is a set of "instructions" that rules the transmission of traffic packets over the Internet). We use two different metrics as proxies for each ISP's traffic flows: one

\footnotetext{
${ }^{1}$ However its impact on the dynamic and productive efficiency is controversial, because of the role of market power as an incentive to innovate.
} 
of these measures infers the number of the ISP's downstream customers, and the other its centrality or "degree of unavoidability".

We assess market concentration in the European upstream Internet market following a two step process: firstly we determine the relevant market applying the vertical and geographical relevant market definition algorithms and criteria; then we calculate market concentration indexes, for the Internet Upstream Routing taking place in Europe, via four leading Internet Exchange Points (IXPs) ${ }^{3}$ : the London Internet Exchange Point (LINX), The Deutsche Commercial Internet Exchange Point (DECIX), the Amsterdam Internet Exchange Point (AMSIX) and the Milan Internet Exchange Point (MIX).

Interestingly, our measures prove to be very close to the one calculated with actual traffic data $^{4}$, indicating a potential application of this approach to the antitrust and regulation authorities guidelines. The results show that some vertical segments of these markets are highly concentrated, while others are extremely competitive. According to the Merger Guidelines [U.S. Department of Justice (1997)], some of the estimated market concentration values would immediately fall within the special attention category.

The rest of the paper is organised as follow: section 2 describes some of the early antitrust inquires for the Internet backbone in the EU and the US. Section 3 discusses the new Regulatory framework for the market of electronic communication issued in 2002 by the Commission and section 4 revises some early related studies. Section 5 describes more recent studies on which we base our classification algorithms while section 6 provides the actual classification, discusses how to use these algorithms to evaluate market concentration across the European IXPs, and suggests how to define the relevant vertical market boundaries. Section 7 applies the vertical and geographical relevant market definition to calculate market concentration in various European Internet upstream markets. Finally section 8 concludes the paper.

\footnotetext{
${ }^{2}$ For a review of this debate see Giovannetti and Ristuccia (2005).

${ }^{3}$ IXPs are independent organizations composed by Internet Service Providers, where they can route their traffic in a cost effective and technically efficient way.

${ }^{4}$ These data were obtained for research purposes from the London Internet Exchange Point under confidentiality and a non disclosure agreement.
} 


\section{Early Antitrust analysis for the backbone market}

The Internet backbone market witnessed an extremely rapid transformation in the last ten years, also because of a large wave of mergers and acquisitions. This process led to growing concerns relating to possible abuse of market power, one expression of which has been identified in the different interconnection charges levied to small and larger providers, a process started in 1997 by UUNET's decision of setting minimum traffic requirements for free peering with smaller ISP's.

An early analysis was provided by Cave (1999) who analyzed the possible problems and/or desirability of having some degree of market power and a hierarchical structure in the Internet backbone.

The two most relevant antitrust cases discussed in the industry have been the merger between MCI and WorldCom in 1998 and the rejected proposed merger between MCIWorldCom and Sprint in 2000. In both cases the identification of the relevant market posed difficult but interesting questions due to the lack of reliable data.

During the 1998 MCI WorldCom merger analysis the European Commission ${ }^{5}$ included in the backbone market all the providers which were able to obtain global connectivity either through private or public peering, needing no transit contracts. This definition was subsequently modified: only the providers reaching global connectivity exclusively via private peering were included in the backbone market (see Buccirossi et al., 2005).

The three relevant markets affected by the proposed merger were identified as being: 1) the provision of host to point of presence connectivity, 2) the provision of Internet access services and 3 ) the provision of top-level or universal connectivity. The investigation, concentrated in this last product market. One of the main issues at stake, and a major source of disagreement between the Commission and the two defendant companies, concerned the hierarchical nature of the Internet. The Commission stressed that a hierarchical structure was clearly exposed by the evidence that top level providers achieve their connectivity entirely by settlement-free peering mainly at private peering points, whereas smaller providers need to purchase transit from top-tier networks to achieve global connectivity ${ }^{6}$. The Commission

\footnotetext{
${ }^{5}$ On the 11th of January 2000, the European Commission received a notification by which MCI-WorldCom would merge with Sprint by an exchange of shares. After an extensive investigation into the merger proposal on the 28th of June 2000 the Commission adopted the decision that " The notified concentration consisting of the merger between MCI-WorldCom and Sprint is declared incompatible with the common market and the functioning of the EEA Agreement." [Official Journal of the European Commission (2000)]

${ }^{6}$ The dominant position of WorldCom had been attained through a very active acquisition policy. In the Civil Action brought by Department of Justice of the United States [U.S. Department of Justice (DoJ) 2000a] against the acquisition of Intermedia Communications by WorldCom are described some of the more than 60 
defined the relevant market as the one composed by the providers equipped with a set of peering agreements with $100 \%$ settlements free connectivity across the Internet and found that only five top-level networks, MCI WorldCom, Sprint, AT\&T, Cable \& Wireless and GTE, satisfied these criteria. Consequently the antitrust authorities defined the market participants as those who peer both with MCI and Sprint and by adding networks accessible directly rather than through a third party, a total of seventeen players were considered for the analysis of the market for top level Internet connectivity. Any other Internet provider, left outside this market definition, would require to purchase transit from at least one of the top five providers to achieve global connectivity.

The proposed new merged entity would have had a market share between [37-51]\% in terms of traffic flows, against the next competitor's one not larger than $15 \%$. The Commission concluded that the proposed merger would have led to the emergence of a top level network provider, able to act almost independently of its competitors and customers and to determine its own, and its competitors, prices and the technical developments in the industry. Another relevant issue, decisive in appraising the competitive effects of the merger, was its effects on potential entry in the industry. Since the peering rules require an entrant to be of considerable size, the Commission found that the merger would have generated a formidable barrier for potential entrants in the top tier backbone market.

Following these considerations, in July 2000, the proposed merger between MCIWorldCom and Sprint has been abandoned after the block imposed not only in the EU but also by the U.S. Department of Justice (DoJ). The Federal Trade Commission considers Market concentration as the fundamental parameter when assessing the competitive impact of a proposed merger. Indeed, following the U.S. Horizontal Merger Guidelines , "A merger is unlikely to create or enhance market power or to facilitate its exercise unless it significantly increases concentration and results in a concentrated market, properly defined and measured. Mergers that either do not significantly increase concentration or do not result in a concentrated market ordinarily require no further analysis."7 The results of the DoJ

acquisitions operated by this company: in 1995 WorldCom acquired the network service operations of Williams Telecommunications in 1996, through the acquisition of MFS Communications Company, WorldCom obtained the control of UUNET, the world largest Internet backbone provider. In 1998 WorldCom acquired Compuserve a leading Internet provider and ANS, AOL's primary Internet backbones network. Other acquired backbones were GridNet, Unicom-Pipex, InNet, NL Net and Metrix Interlink. As a result of the leadership position reached in these years the WorldCom acquisition of MCI in September 1998 has been accompanied by the imposition, by the US DoJ and the EU Commission, for MCI to divest its Internet assets to Cable \& Wireless.

7 In detail, the FTC uses the Hirschman-Herfindahl Index ("HHI") of market concentration. The HHI is calculated by summing the squares of the individual market shares of all the participants and multiplied by ten 
merger analysis found that "the proposed merger of WorldCom and Sprint will cause significant harm to competition in many of the nation's most important telecommunications markets. By combining two of the largest telecommunications firms in these markets, the proposed acquisition would substantially lessen competition in violation of Section 7 of the Clayton Act... For millions of residential and business consumers throughout the nation, the merger will lead to higher prices, lower service quality, and less innovation than would be the case absent its consummation. The United States therefore seeks an order permanently enjoining the merger." This motivation was based on the role of backbone market concentration as expressed by the HHI, calculated on the traffic shares, which was, before the merger, approximately 1850; and it would have risen, because of the merger, by approximately 1150 points to circa 3000. [U.S. Department of Justice (DoJ) 2000b] ${ }^{8}$.

\section{New Regulatory Framework and the Commission's guidelines}

The regulatory interest in the backbone market remained high and, after a public consultation on the Review of the Electronic Communications Sector, the Commission proposed in July 2000 a package of measures introducing a new regulatory framework for electronic communication networks and services. This was intended to provide a lighter regulatory touch where markets have become more competitive while supporting sustainable and affordable prices and protecting basic consumers rights ${ }^{9}$. The application of the Regulatory framework was then essentially described, also in its relevant operational aspects, in the "Guidelines on market analysis and assessment of significant market power under the Community regulatory framework for electronic communications networks and services" published on the $11^{\text {th }}$ of July 2002 .

The Commission's guidelines focus only on issues related to (i) market definition; and (ii) the assessment of significant market power (SMP) within the meaning of Article 14 of the

\footnotetext{
thousands. The guidelines focus on two figures: pre-merger HHI concentration index level, and post-merger HHI concentration increments. When the post-merger HHI is below 1000, the guidelines consider the market as being non concentrated, and no further analysis is required. When the post-merger HHI ranges between 1000 and 1800 the guidelines consider these markets as moderately concentrated. In this case further scrutiny is required only if the merger would increase the HHI of more than 100 points. Finally, when post-merger HHI is above 1800 the markets are considered highly concentrated. In this case an increase in the HHI of more than 50 points will start raising concerns, while an increase of more than 100 points is considered as potentially dangerous for the increase of market power it will entail.

${ }^{8}$ http://www.usdoj.gov/atr/cases/f5000/5051.pdf

9 The final test was published on the $4^{\text {th }}$ of February 2002, (See Official Journal 2002 (http://europa.eu.int/information_society/topics/telecoms/regulatory/new_rf/documents/03672en1.pdf ).
} 
framework Directive, that individuated SMP when a firm "enjoys a position of economic strength affording it the power to behave to an appreciable extent independently of its competitors, customers and ultimately consumers".

\section{Market Definition: Assessing Vertical Market Boundaries}

The concept of relevant market is a key issue for antitrust analysis, since it is central to the assessment of market power. Indeed, article 81 in the Treaty of Rome, states that the limitation of competition is to be assessed on the relevant market; while the article 82 defines market dominance in relation to market shares, and the merger control is based on the dominant position in relation to market shares.

Among different market definitions, two have received most attention from the literature. One approach focussed on the "economic markets", market for goods resembling each other where the law of one price was supposed to operate. However Werden and Froeb (1994) and Scheffman and Spiller (1987) argued that this concept of economic market was inadequate to antitrust analysis. The aim of the second approach was to define the relevant market as instrumental to its applications in the antitrust analysis. The leading definition of relevant market, adopted by the European Commission, hence, is based on the characteristics of substitutability among products, expressed by the cross-price elasticities: the relevant market is the set of products and geographic regions which in some way constrain the pricing behaviour of the firms providing the product under scrutiny; this means that the relevant market is the set of commodities which are, in respect to consumers' preferences, good substitutes of each other, so that a price change in one of them will provoke a demand change in the other.

Whenever assessing whether or not a given set of products or services in a given geographical area constitutes a relevant market, for competition policy purposes, one has therefore to assess the existence, and strength, of competitive constraints on the price-setting behaviour of the producers of this set of commodities. A relevant market is then composed by the set including all of the commodities for which, if taken as a whole, the excluded ones do not have competitive effects on them. These competitive constraints can either arise from the demand-side of the market through demand substitution of the commodities under analysis or from the supply-side substitution, either through existing or potential 
competitors $^{10}$. Supply-side substitutability indicates whether suppliers other than those offering the commodity in question start offering the relevant commodities, themselves, or whether they react to the initial price increase by changing the price of their related commodities.

The usual, empirical way of assessing demand and supply-side substitution is to apply the so-called 'hypothetical monopolist test'. This test asks what would happen if there were a small but significant, lasting increase in the price of a given product or service, assuming that the prices of all other products or services remain constant. This test is also known as 'SSNIP' (small but significant non transitory increase in price) and its importance lies primarily in its use as a conceptual tool for assessing evidence of competition, based on substitutability, between different products or services.

The nature of the SSNIP test is recursive. It starts by considering an initial set of products that are thought to define the market and simulate an increase in their price; in practice, the Commission's guidelines suggest that the National Regulatory Authorities should normally consider reactions to a permanent price increase of between 5 and $10 \%$. Suppose that the price increase is unprofitable, since consumers are substituting other products for the one the price of which increased: in this case the test has to be re-carried, with reference to the set of commodities composed by the initial one and by all those other commodities which the test found as relevant substitutes in its previous rounds.

The SSNIP test should be repeated considering an increasing set of products up to the point where a relative price increase within the geographic and product markets defined will not lead consumers to switch to readily available substitutes or to suppliers located in other areas

The SSNIP approach has however some limitation. In particular, one of the identified problems is that the test outcome depends on the initial price level considered. Indeed, apart from the special case of a constant price elasticity demand function, the size of the demand reaction to a price increase will necessarily depend on the existing price level. If this starting price level is already at the Monopoly level, (the optimal price in terms of profit maximization), then any further price increase will lead to a profit loss. In these circumstances, where a firm has already exercised market power, a situation known as the 'cellophane fallacy', the SSNIP test would lead to a larger market extension than in the case were initial prices were set at a competitive level.

${ }^{10}$ Clearly potential competitors substitution will take a longer time interval than the existing competitors' one. 
One further problem in applying the SSNIP test arises when there are forms of discrimination, which generates separate markets for the same commodity, depending on the customer's characteristics. Discrimination is however an essential feature characterising Internet Interdomain routing, our object of study. Indeed, as we argued before, interconnection agreements usually take the form of transit or peering. While transit is a contractual relation rife with discrimination practices, starting from its typical non-linear pricing structure, peering is a bilateral interconnection decision based exclusively on discrimination and formally expressed in the peering policy of every provider.

Finally another major limitation lies in the data unavailability to perform the SSNIP Test. Again, this is particularly true in the Upstream Internet routing, and further motivates us in the construction of price-independent market structure indicators.

\section{Supplying Universal Connectivity}

Final users express a demand for Internet Connectivity, and ISPs's role is to supply it. This implies generating both incoming traffic, by demanding contents stored at a given off-net location, and outgoing traffic exporting contents stored in its own routes. To supply this service ISPs will need to be able to cover the total set of IP addresses. Universal connectivity is indeed the ISPs' production output. The inputs required to produce this output will be three: 1) ISP's own routes, and Off-net routes accessed: 2) through peering agreements and 3) though transit agreements via an upstream provider. In this setting the problem of market definition should be assessed in terms on demand elasticities for input factor, i.e, the traditional role played by consumer's preferences and their cross-elasticities of substitution is now played by ISPs and their input demand functions cross-elasticities.

In this setting, a change in relative input prices, for example a reduction in transit prices, will modify the initial input demand to a different ratio between transit and peering ${ }^{11}$. However, this traditional microeconomic approach is now inapplicable since the technological decision is constrained by the number, and identity, of willingly peering partners. This implies that, while a Small but Significant Non-transitory Increase in Price for transit might induce an ISP to substitute some of its existing Transit routes with new Peering ones, it might well be that this switch will be constrained by the unwillingness of the

\footnotetext{
11 In Economics, it is usually assumed that the exact combination of inputs, in our case peering and transit agreements, is derived by a cost minimizing choice conditional to a given level of output. This depends, of course, upon the functional form of the production function describing the technology, which uses these inputs (transit and peering agreements) to produce the ISP output: Universal connectivity.
} 
other ISPs to peer with it. These difficulties led us to introduce a market segmentation algorithm (developed in Section 5), based on a classification of ISPs which reflects the existence of bilateral peering refusals.

\section{Market Definition: Assessing Geographic market Boundaries}

Traditionally, the process of defining the boundaries of the geographic market proceeds along the same lines as those discussed above in relation to the assessment of the demand and supply-side substitution in response to a relative price increase. In the electronic communications sector, the European Commission guidelines indicate two main criteria to determine the geographical scope of the relevant market: (a) the area covered by a network; (b) the existence of legal and other regulatory instruments. On the basis of these criteria, geographic markets can be considered to be local, regional, national or covering territories of two or more countries. For the specific market of Internet upstream access in particular, linguistic differences should play a minor role in segmenting the geography of Interconnection. The European Commission guidelines also state that the relevant geographic market comprises an area in which the undertakings concerned are involved in the supply and demand of the relevant products or services, and the conditions of competition are similar or sufficiently homogeneous; moreover the area must be distinguished from neighbouring areas in which the prevailing conditions of competition are appreciably different.

Once again, the application of these criteria to the Internet upstream market seems at least problematic. Hence, in this paper we follow a different approach to define the geographical borders of the Internet Upstream Market. We first start by looking at the features characterising the European IXPs in terms of the distribution of their members within the different hierarchies of the Internet. Universal connectivity in the Internet is achieved through the interconnection between all the hierarchies in the Internet, from the very bottom (end users and Internet Access Providers, or IAP) to the very top (Tier-1 providers), through the middle hierarchies. An ISP in the middle of the Internet hierarchy needs to be connected to at least one Tier-1 to provide universal connectivity. Hence, two different IXPs will not be considered as geographically separated markets if one IXP is characterized, for example, by the presence of Tier 1 providers while the other IXP does not have Tier 1 among its members. Indeed, in this case the two IXPs could complement each other, and therefore the market is not geographically separated. In particular, we will consider as independent locations the area having an IXP, that can provide Universal connectivity through the 
presence of at least one Tier-1 Member. In conclusion, contrary to the guidelines suggestions, similarity in competition implies duplication of access modalities at different locations, indicating therefore geographically separated markets, while structural differences amongst IXPs will indicate, through access complementarities, a single geographical market.

\section{Significant Market Power}

Once the market definition problem has been addressed, the next step is to assess the existence of significant market power. According to Article 14 of the framework Directive ${ }^{12}$, an ISP is "deemed to have significant market power if, either individually or jointly with others, it enjoys a position equivalent to dominance, that is to say a position of economic strength affording it the power to behave to an appreciable extent independently of competitors customers and ultimately consumers."

Often, the lack of evidence or of records of past behaviour or conduct will mean that the market analysis will have to be based mainly on a prospective assessment. In these cases, a dominant position is found by reference to a number of criteria and its assessment is usually based on existing market conditions; in particular, market shares are often used as a proxy for market power. Although a high market share alone is not sufficient to establish the possession of significant market power (dominance), it is unlikely that a firm without a significant share of the relevant market would be in a dominant position. Thus, the guidelines stress that firms with market shares of no more than $25 \%$ are not likely to enjoy a (single) dominant position on the market concerned. In the Commission's decision making practice, single dominance concerns normally arise in the case of firms with market shares of over $40 \%$, although the Commission may, in some cases, have concerns about dominance even with lower market shares ${ }^{13}$. Concerning the methods used for measuring market size and market shares, the Commission's guidelines state that both volume sales and value sales provide useful information for market measurement ${ }^{14}$. These data are however usually unavailable for the upstream Internet connectivity market.

\footnotetext{
12 This is the definition that the Court of Justice case-law ascribes to the concept of dominant position in Article 82 of the Treaty of Rome.

13 According to established case-law, very large market shares — in excess of $50 \%$ — are in themselves evidence of the existence of a dominant position.

${ }^{14}$ In particular, in the case of bulk products, preference is given to volume, whereas in the case of differentiated products (i.e. branded products), sales in value and their associated market share will often be considered to reflect better the relative position and strength of each provider.
} 


\section{Exploring of the backbone through Cyber-geography: Early Studies}

The analysis of the Internet Upstream Routing, understanding its boundaries, traffic flows, prices, market shares and revenues is particularly challenging both for the lack of satisfactory statistical data and for the elusiveness, due to the non-dedicated connection modes of the Internet Protocols, of the traffic exchanged among operators.

The public nature of the Internet and the routing protocols on which it is based often allow, however, the analysis of the paths followed by information packets from origin to destination through the Internet. An entire branch of research, Cybergeography, is devoted to the mapping of this physical-virtual world. The Cooperative Association for Internet Data Analysis, (CAIDA) constructed a global Internet topology focussing on measuring the performance of specific paths through the Internet. Claffy et al. (1999), using samples covering 20,588 end destinations, determined the frequency with which an individual backbone provider (identified by an Autonomous system number, AS) appeared in a path and the relative depth of those appearances, both in terms of number of backbones and the number of hops crossed from the source. In their findings, CerfNet/AT\&T, Cable \& Wireless (which purchased Internet MCI's backbone in 1998), Sprint, and UUNET played a major role in transporting packets across the Internet. Cossa (2000) considered a dataset from Boardwatch magazine Internet Service Providers Directory, 1999's edition, showing the breakdown of 8,950 backbone connections from 5,078 Internet service providers per major backbone. With these data she evaluated the impact of the MCI WorlCom- Sprint merger in terms of market concentration. Cossa also calculated the HHI based on the number of upstream backbone connections and showed that the pre-merger HHI increased from 1450 up to 2090 as a result of a merger between the two companies. In the next sections we describe more recent algorithms and concepts as we will use them to construct Market concentration indicators for the European Internet Upstream Routing Market.

\section{Recent studies on how to infer the Economic Relationship between two ISPs}

A growing body of literature in the networking community works on defining the economic position of an ISP, by evaluating the type of relationships it has with other ISPs. Since the business part of this relationship is decided at a bilateral level and kept private, one has to infer the type of relationship from the network connectivity structure resulting from the 
available data on inter ISP interconnection or Upstream routing. In this section we briefly describe datasets and algorithms used to explore this inter-ISPs connectivity structures, which we will then utilize to assess market concentration.

\section{Actual Internet Routing}

The main part of the actual Internet traffic exchanges (routing) happens at IP routers. These have a table, whose role is to match an IP address contained in the header of a data packet to the link leaving the router in the right direction. Through these tables each Autonomous System - or AS- (ASes are Internet operators consisting in either a single network or a group of networks that is controlled by a common network administrator) announces, via the Border Gateway Protocol (BGP), to a neighboring AS a list of paths made of more AS-nodes leading to a final destination AS. The implementation of the routing policy determines which BGP information in an ISP is generated, passed on and to whom of the connected neighbors: which path is being presented.

The interplay of all the ASes individual routing policies results in the global connectivity map for data transmissions across the Internet. This paper is motivated by the belief that no reliable empirical alternative exists to this microrouting analysis for the study of the upstream Internet market structure, and for its antitrust analysis.

\section{Business Relationships}

There are three ${ }^{15}$ basic types of business relationships that shape ISPs Upstream routing policies: Customer-Provider, Provider-Customer and Peer-Peer. It is widely assumed in the current literature that these three types of business relationships sufficiently capture various contracts and agreements arising in the Internet. Although this is an oversimplification, the basic business relationships provide a relatively close approximation in practice. These basic types of business relationships are explained as follows:

Customer-Provider/Provider-Customer: in this relations both parties assume asymmetric roles; the provider sells the reachability of IP addresses, the ability to transit all traffic to any destination, while the customer pays for this connectivity.

Peer-Peer: usually, both parties exchange the traffic for their customers only - routes to their providers and other peers are not revealed to each other. This is because peer-peer

\footnotetext{
${ }^{15}$ The Computer Science Literature also considers a fourth type of relation, among Siblings, where both AS belong to the same ISP. The ISP partitions his network to ease the technical management by hiding internal information for each of the separate AS.
} 
connectivity is usually not paid, and there is no incentive to share one's other paid connectivity.

\section{Type of Relationship Inference from BGP Graphs}

In this paper we suggest that the most useful way to learn about the economic type of relationship between two ASes is by examining BGP dataset. Some ASes publish their BGP path tables, and from this collection of paths it is possible to derive a network graph of the Internet that describes the connectivity at the AS level. In the analysis of the BGP path tables, one central assumption, first formulated by Gao (L. Gao, 2001), is made to infer relationship types. This assumption states that all paths are free of relationship-valleys. Intuitively speaking, one can imagine a particular path to describe the trail of ISPs that an IP data packet has to traverse to reach its destination network. This path of ASes will start at an ISP who is a customer of the next "upstream" provider of IP connectivity, who in turn is a customer of the next provider. Following this chain of customers, at some point we will reach the peak in the hierarchy of AS that participate in this path, and from there on we expect the IP data packet to descend a chain of provider to customer relations between ISPs till it reaches the ultimate destination. The important observation in this description is that in any path there is only one consecutive chain of upstream and one consecutive chain of downstream ISPs present. Figuratively speaking, we assume that there are no valleys in these hills of upstream/downstream chains. The following figure shows a set of valid paths and an invalid valley.

\section{Figure 1: Examples of valid paths}

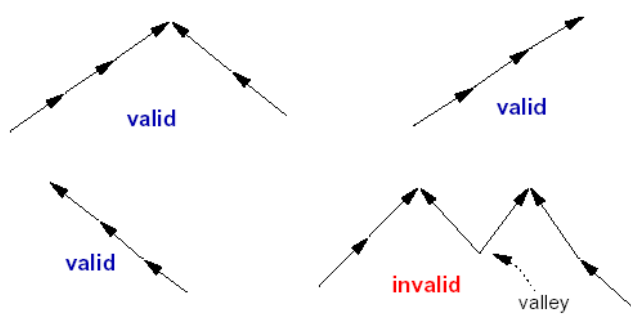

The intuition behind this assumption is that such a valley would imply that a customer is transferring traffic from one of his providers to another provider and pays the first one for receiving the traffic and the second to have it forwarded to the destination. Since doing so would be economically irrational, we can assume that occurrences of such routing policy 
patterns are mis-configurations and any ISP has great incentive to rectify this situation quickly.

The next assumption about AS path constraints states that peering is not transitive. Hence for each AS path, there is only one peering link possible, and this can only be at the peak of the path, exactly between the upstream and the downstream AS chains. This constraint follows from the idea that the only traffic an ISP would accept from a peer is the traffic from that peer and the one of his customers. If an ISP were to accept traffic from the providers of a peer, then the ISP would actually perform a transit function for the providers of the peer. Since nobody pays the ISP for this transit traffic, we can expect the ISPs to refuse such traffic by filtering out routes that a peer might advertise with destinations that are not contained in his AS, or his set of customers (not only the immediate customers, but all the further customers down the line as well).

\section{Internet Service Providers Classification}

Historically, it was common to classify ISPs into a strict hierarchy of Tiers, whereby the ISPs within one tier were considered equally relevant in terms of network transport capacity and economic bargaining power. In the following sections we will use a ASes classification derived from the ranking obtained from CAIDA's AS-relationship inference/ranking algorithm. Using the inferred relationships, a ranking is derived based on the dimension of the set of customers of each AS. Since the relationship inference algorithm relies on valleyfree path relationships, we can assume that in the direction of a customer, down to the end of an AS path, all ASes are themselves customers of the previous AS. This leads to a tree, or "customer cone", as it is referred to by Dimitropoulos et. al(2005) containing the set of customers, including all the customers of these. The ASes ${ }^{16}$ are then ranked, based on the dimension of this customer cone.

\footnotetext{
${ }^{16}$ For technical reasons, large ISPs operate using multiple ASes (e.g. UUNET uses 13), and it would be a misrepresentation to rank each of these ASes separately, since they belong to the same company. The CAIDA ranking aggregates ASes with company names that are similar in the ARIN database (two names are considered similar, if they are identical, except for the last several characters). We rely on this grouping to consider rankings of ISPs, instead of rankings of ASes only, since it improves the representation of the ranking, despite some shortcomings.
} 


\section{Vertical Boundaries and Market Concentration within the Euro-IX Members}

\section{Data description}

IXPs $^{17}$ are independent organizations composed by Internet Service Providers, where they can route their Upstream traffic in a cost effective and technically efficient way.

The data used for the empirical analysis were collected in subsequent steps. Firstly we obtained the lists of ISPs members for each Internet Exchange Point participating at the Trade Association Euro-IX ${ }^{18}$. Then, for each IXP, and for each IXP's member, we obtained two sets of measures useful to assess its position within the Internet: the first metrics, provided by CAIDA, associates a rank to each AS by looking at their location in the Internet hierarchy. The rank is derived from the AS customer cone, defined as the AS itself plus its customers, plus its customers' customers, and so on ${ }^{19}$. We then used the algorithm devised by Huber et al (2004) to infer the relationships between pair of ASes (provider to customer, customer to provider, peering, sibling, no relationship) within each IXPs.

The second metrics is derived from the BGP tables displayed by Oregon Routeviews Project $^{20}$. Following Shimbel (1953) we calculated a measure of betweenness (centrality) for each AS $v$ (see Brandes, 2001 for a survey on the algorithms used to compute betweenness). In particular, the betweenness for AS $v$ is given by the number of BGP paths - between any pair of ASes - that traverse $v$. Formally,

$$
B_{s}(v)=\sum_{s \neq v \neq t \in V} \sigma_{s t}(v)
$$

where $\sigma_{s t}(v)=\sigma_{t s}(v)$ is the number of shortest paths from the AS $s$ to the AS $t$ on which the AS $v$ lies on. High betweenness indicates both that an AS node can reach other ASes on

\footnotetext{
${ }^{17}$ IXPs play an absolutely crucial role in the Internet traffic Routing. As an example it is sufficient to think that more than $90 \%$ of the Internet UK traffic is routed through the LINX-IX in London.

${ }^{18}$ AIX, AMS-IX, BCIX, BIX, BNIX, CATNIX, CIXP, DE-CIX, Equinix * +, ESPANIX, FICIX, GIGAPIX, GN-IX, INEX, JPNAP * +, LINX, LIPEX, LIX, LoNAP, MaNAP, MIX, MSK-IX, NaMeX, NDIX, Netnod *, NIX, NIX.CZ, NOTA +, PARIX, RoNIX, SIX, TIX, TOPIX, VIX, XchangePoint *, where* indicates IXPs with multiple unconnected locations and + indicates IXPs located outside of Europe.

${ }^{19}$ The customer cone can be defined using three different precision levels: the AS cone, the AS prefix customer cone, the AS /24 prefix customer cone. The AS cone indicates the size of the customer cone in terms of number of ASes; this is a rough measure, since individual AS sizes can be very different. Since each AS advertises a different number of prefixes, and the smallest bit of a prefix is the $/ 24$, the others two measures provide greater accuracy in assessing the size of the customer cone. For full details see the CAIDA relevant web page at
}

(http://www.caida.org/analysis/topology/rank_as/index.xml). 
relatively short paths, and that this AS has a certain degree of market power over the others, since it may be costly to avoid the central ASes and follow other paths in order to deliver packets over the Internet. This parameter clearly captures and measures the economic concept of partial essential facility for central ASes. We calculated the betweenness, for every AS, with respect to the population of other ASes of the IXP it is a member of. This means that the betweenness we calculate is defined only by looking at the paths involving ISPs members for each IXP; hence, it provides additional useful information on the partial bottlenecks, centrality of given members within an IXP and not within the Internet as a whole.

\section{Market Power and Market Concentration}

The most widely used measure of market power enjoyed by a certain firm $i$ operating in the market $M$ is provided by the Lerner index $L_{i}=\frac{m_{i}}{\varepsilon}$, where $m_{i}$ is the market share of firm $i$ and $\varepsilon$ is the market elasticity of demand. The index of overall market power is then given by

$$
L=\sum_{i} m_{i} L_{i}=\sum_{i} \frac{m_{i}^{2}}{\varepsilon}=\frac{H H I}{\varepsilon}
$$

where $H H I=\sum_{i} m_{i}^{2}$ is the Herfindahl-Hirschman Index of concentration.

From (2) it is clear that the HHI, and hence the pattern of market shares will provide useful insights to assess the degree of market power. Our unit of analysis is again the Internet Exchange Point, where a large share of Upstream Internet Routing is exchanged.

We devised two different proxies ${ }^{21}$ for the market share $m_{i}$ :

- The first measure is directly obtained from the customer cone metrics: the market share for each AS is obtained as the ratio of its customer cone and the sum of all the customer cones of the IXP members.

- The second measure is derived from the betweenness of an AS. In particular, the proxy for firm $i$ 's market share is obtained comparing its relative betweenness, or degree of unavoidability, within the IXP: this is computed by dividing the betweenness value for the AS $i$ by the sum of the betweenness values for all the ASes considered. Formally,

\footnotetext{
${ }^{20}$ For the full details, see the University of Oregon routeviews project webpage at http://www.routeviews.org/

${ }^{21}$ Ideally, market shares within the IXP are derived by looking at the traffic flows. Since these data are confidential we use the metrics introduced in the previous sections.
} 


$$
\operatorname{Rel}_{S}(v)=\frac{B_{S}(v)}{\sum_{v} B_{S}(v)}
$$

where $B_{s}(v)$ is defined in (1).

This last measure focuses on the presence of essential facilities, often the root cause of the presence of market power. In particular, the Internet is ambivalent about the presence of essential facilities: its nature, the protocols which define the routing procedures, are indeed meant to avoid predefined paths, making therefore easier to avoid essential facilities or bottlenecks. However how successful traffic routing is in avoiding bottlenecks depends essentially on the design of the interconnections among ISPs, and on its hierarchical structure. Usually understanding the presence of an essential facility requires an assessment of how easy it is to duplicate a given input. In the specific context of the Internet, this means an assessment of how easy is it to bypass a given route, or a node, managed by an AS that refuses peering and requires a transit charge, a paid input. The betweenness parameter expressly captures the presence of partially essential facilities in Internet outing by focussing on how, avoidable or non avoidable, certain nodes are.

A first question that needs to be addressed is the following: are these proxies a reasonable approximation of the effective market share within the IXP? In order to address this question, we obtained confidential traffic data at LINX for the period October 2004November 2004. We then calculated the market share for each LINX member using both inbound traffic and outbound traffic; hence we computed the HHI index, finding a very strong correspondence with the HHI index calculated using the CAIDA customer cone metrics $^{22}$.

There is no immediate sensible comparison between the market share calculated by the CAIDA rank and the one calculated using the betweenness data. Indeed, while the first is targeted to capture the market share in terms of the established market position and, more explicitly, the pattern of traffic flows, the second is meant to capture the relevance within the IXP from an essential facility point of view. Table 1, below, shows the calculations for the

\footnotetext{
${ }^{22}$ : 0.021 is the effective HHI, while the indexes calculated with CAIDA customer cone, and betweenness are 0.024 , and 0.05 respectively. Moreover, the market share squares calculated with the CAIDA customer cone showed the highest correlation (0.74) with the squares of effectives market shares, (the correlation is instead 0.33 for the betweenness based proxies). This is a positive result indicating that it is sensible to calculate the $\mathrm{HHI}$ index by using the CAIDA rank-based proxy market shares, and also that these proxies are probably a good approximation of the effective market shares.
} 
two concentration measures for all IXPs under analysis. We ranked the different IXPs according to their CAIDA customer cone metrics-related HHI.

Table 1: IXPs Concentration Indexes

\begin{tabular}{|c|c|c|c|c|}
\hline ixp & Location & size & hhi (c_c) & hhi (betweenness) \\
\hline$\overline{a m s-i x}$ & Amsterdam (NL) & 221 & 0.025 & 0.051 \\
\hline $\operatorname{lin} x$ & London (UK) & 179 & 0.024 & 0.046 \\
\hline dec-ix & Frankfurt (GER) & 145 & 0.033 & 0.067 \\
\hline equinix & 7 locations (US) & 187 & 0.023 & 0.048 \\
\hline nota & Miami (US) & 96 & 0.041 & 0.069 \\
\hline netnod & Stockholm (SWE) & 46 & 0.102 & 0.118 \\
\hline parix & Paris (FR) & 39 & 0.056 & 0.097 \\
\hline bnix & Brussels (BE) & 47 & 0.092 & 0.210 \\
\hline$v i x$ & Vienna (AUS) & 82 & 0.071 & 0.100 \\
\hline xchangepoint & London $(U K)$ & 128 & 0.059 & 0.094 \\
\hline namex & Rome (IT) & 19 & 0.489 & 0.236 \\
\hline lipex & London (UK) & 53 & 0.986 & 0.069 \\
\hline tix & Zurich $(\mathrm{CH})$ & 51 & 0.083 & 0.114 \\
\hline $\operatorname{mix}$ & Milan $(I T)$ & 57 & 0.083 & 0.190 \\
\hline bcix & $\operatorname{Berlin}(G E R)$ & 26 & 0.493 & 0.424 \\
\hline$n i x$ & Oslo (NORWAY) & 59 & 0.121 & 0.143 \\
\hline$n d i x$ & Enschede (NL) & 11 & 0.997 & 0.620 \\
\hline in-ex & Dublin (IRL) & 18 & 0.482 & 0.757 \\
\hline$g n-i x$ & Groningen $(N L)$ & 28 & 0.239 & 0.166 \\
\hline gigapix & Lisbon (PORT) & 21 & 0.501 & 0.448 \\
\hline $\operatorname{cixp}$ & Geneva $(\mathrm{CH})$ & 24 & 0.143 & 0.188 \\
\hline$m s k-i x$ & Moskow (RUSS) & 156 & 0.179 & 0.259 \\
\hline $\operatorname{six}$ & Ljubljana (SLOVE) & 11 & 0.193 & 0.232 \\
\hline espanix & Madrid (SPA) & 28 & 0.125 & 0.172 \\
\hline aix & Athens (GRE) & 15 & 0.996 & 0.170 \\
\hline nix.cz & Prague (CZ) & 55 & 0.125 & 0.159 \\
\hline manap & Manchester (UK) & 29 & 0.248 & 0.123 \\
\hline catnix & Barcelona (SPA) & 20 & 0.494 & 0.259 \\
\hline ronix & Bucharest (ROM) & 21 & 0.219 & 0.266 \\
\hline lonap & London (UK) & 43 & 0.971 & 0.128 \\
\hline ficix & Helsinki (FIN) & 21 & 0.237 & 0.243 \\
\hline topix & Turin (IT) & 20 & 0.943 & 0.328 \\
\hline jpnap & Tokyo (JAP) & 48 & 0.110 & 0.187 \\
\hline bix & Budapest (HUNG) & 54 & 0.243 & 0.127 \\
\hline lix & Luxembourg $(L U X)$ & 12 & 0.204 & 0.353 \\
\hline
\end{tabular}


According to HHI index using the CAIDA rank-based proxy market shares the fifteen least concentrated IXPs are AMS-IX, LINX, DE-CIX, EQUINIX, NOTA, NETNOD, PARIX, BNIX, VIX, EXCHANGEPOINT, NAMEX, LIPEX, TIX, MIX AND BCIX. This order, as we can see from table 2, is not reflected when the HHI concentration index is calculated according to the betweenness.

Table 2: Cross ranking top 15

\begin{tabular}{|c|c|}
\hline $\begin{array}{l}15 \text { least } \\
\text { concentrated HHI cc }\end{array}$ & $\begin{array}{l}15 \text { least } \\
\text { concentrated HHI } \\
\text { betweenness }\end{array}$ \\
\hline 1. $\quad$ ams-ix & 1. $\operatorname{lin} \mathrm{x}$ \\
\hline 2. $\quad \operatorname{lin} x \quad \ldots$ & 2. equinix \\
\hline 3. dec-ix ......... & 3. ams-ix \\
\hline 4. equinix & 4. dec-ix \\
\hline 5. nota & 5. nota \\
\hline 6. netnod & 6. lipex \\
\hline 7. parix & $7 \%$ exchangepoint \\
\hline 8. bnix & 8.7 parix \\
\hline 9. vix & 9. vix \\
\hline 10. exchangepoint & 10. tix \\
\hline 11. namex & 11. netnod \\
\hline 12. lipex & 12. manap \\
\hline 13. tix & 13. bix \\
\hline 14. $\operatorname{mix}$ & 14. lonap \\
\hline 15. bcix & 15. nix \\
\hline
\end{tabular}

These ranking asymmetries are important in showing the different aspects of concentration captured by the two complementary proposed indexes. This is natural for the antitrust analysis of complex network industries where concentration can only be captured along different dimensions, in this case customer base, and network centrality.

In this first step of the analysis we considered the entire memberships of the IXP as if they were part of the same market and calculated the newly proposed concentration indexes accordingly. This is clearly not the case given the difference in ranking amongst the ASes member s of any given IXP. In the next section we address this problem by introducing the vertical market boundaries within these memberships. 


\section{Vertical Boundaries}

In this section we use the CAIDA rank measure, introduced before, to derive the ASes' distribution over a set of vertically separated classes, by looking at jumps in their customer cones values. In particular, we group all ASes into four major groups according to their CAIDA ranking reported below:

a) "Tier 1" (Rank 1-12): this set contains the ISPs that are located at the top of the Internet hierarchy. Most of the providers in this class are the so called Internet backbones of Tier 1 . Tier 1 providers distinguish themselves from any other ISP, by not paying for transit traffic to any other ISP. They only have peers and customers. To achieve global path reachability they peer with other ISPs in this class ${ }^{23}$.

b) Core composed by those ASes ranking between 13 and 250. This group can be subdivided into two sub groups:

i) "Inner core" (Rank 13 - 49): most of the ISPs included in this class need to buy transit from one or more tier 1 ISPs to reach all paths, but only from those, and are able to contain a large proportion of their traffic within their own and their customers' networks. This set contains many important ISPs, such as Korea Telecom, France Telecom, Tiscali. It also contains the largest university, California State University (a network of dozens of campuses); and

ii) "Outer Core"(Rank 50 - 250): this set contains many large players, who are not transit providers, such as HP, Microsoft, Apple, but also significant ISPs such as Peer 1 Network Inc., Hutchison Global Communications, CHINA UNICOM, Bell South.net, as well as large academic networks such as the ones of the UK, Germany and China. A few universities with the largest address space allocations also fall into this class, such as Harvard and MIT $^{24}$.

c) Transit composed by those ranking between 251 and 4000 ; this group can be divided into three sub groups:

\footnotetext{
${ }^{23} 1$ This class actually contains about 18 known ISPs, but in the CAIDA ranking, several of these ISPs are ranked significantly below number 12 (e.g. British Telecom = rank 36 and AOL Transit Data Network = rank 48).

${ }^{24}$ The \#/24-number in the customer cone drops by over one order of a magnitude within this class.
} 
i) Regional Transit(Rank 251 - 1000): this set contains many regionally relevant ISPs, such as Road Runner (US), Telecom Argentina S.A, Nextra Austria, Asia Online New Zealand, States of Michigan, Georgia, Arkansas, Minnesota, etc. Some larger universities can also be found here, such as The University of Texas at Austin, Stanford, and the University of British Columbia ${ }^{25}$.

ii) Local Transit (Rank 1001 - 2500): the ISPs listed in this set contains many locally relevant transit players, e.g. Boston Data Centers Inc., OmanTel, Tiscali Belgium, Portland - Metro Area Network (P-MAN), ARBINETTHEXCHANGE, INC., Danish network for Research and Education, many universities, such as Yale, Emory, University of Virginia, and the University of New Mexico. The \#/24-number in the customer cone drops by $72 \%$ within this class.

iii) Campus Level(Rank 2501 - 4000): this class contains many corporate campus level network (e.g. Cray Inc., Wachovia Operational Services Corporation, Morgan Stanley Dean Witter, BASF Corporation, Oracle Corporation Datacenter, Wal-Mart Stores, Inc.) as well as many university campus (e.g. Mount Sinai School of Medicine, Bradley University, Brigham Young University, Bates College, Georgia State University, University of Salzburg), networks and a few smaller ISPs with local reach (e.g. China Information Broadcast Network Ltd.Co., China Enterprise Communications Ltd., ADC Telecommunications Inc., Skyrr ISP Network $)^{26}$. And finally we have the

d) ISP customers (Rank below 4000). Most of these ISPs do not have any further customers, are leafs in the hierarchy. Some of these ISPs, do have customers, up to one dozen, but are more likely to have between 1-5 customers, if they have any. The networks grouped in this list are many small customer ISPs (e.g. BusinessOnline AG German ISP, Wave2Wave Communications, Inc., FreiNet GmbH, Pacific Information Exchange, Inc., Kabel Deutschland Breitband Service GmbH, Belize Telecommunications Limited, Startec Global Communications, TSI Telecommunication Services, Northeast Telecom Inc.), a few companies (e.g. DuPont, First Citizens Bank, Hotels.com, Deloitte Consulting) and some universities,

\footnotetext{
${ }^{25}$ The \#/24-number in the customer cone drops by over one order of a magnitude within this class.

26 The \#/24-number in the customer cone drops by a further $63 \%$ within this class
} 
schools and public institutions (e.g. University of the Aegean, Innsbrucker Kommunalbetriebe AG, National Dong Hwa University, The Open University of Hong Kong, University of Tehran).

In the following figures we are able to show the vertical hierarchical composition for all IXPs members of the Euro-IX, by classifying their ISPs members into one of the four categories introduced above.

Figure 2.a: IXPs class composition

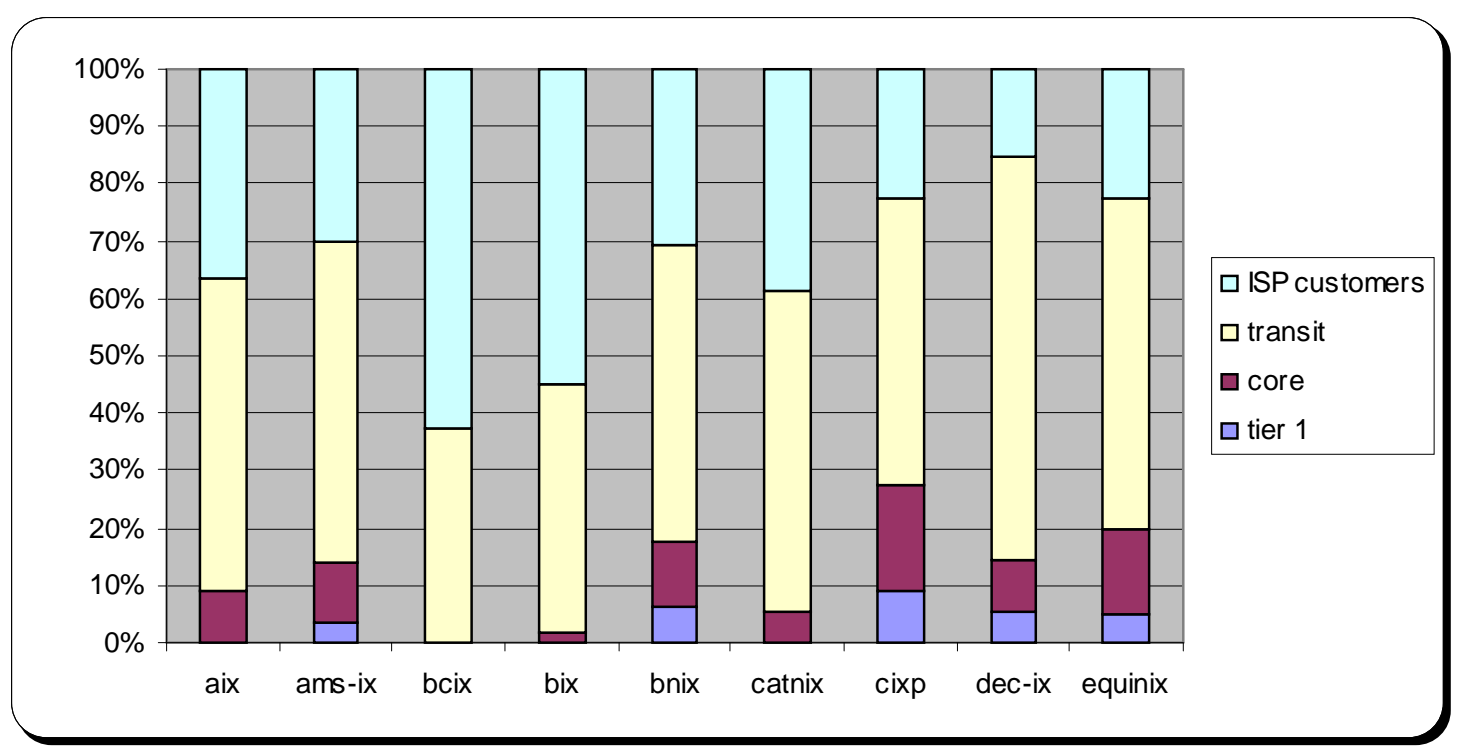

Figure 2.b: IXPs class composition

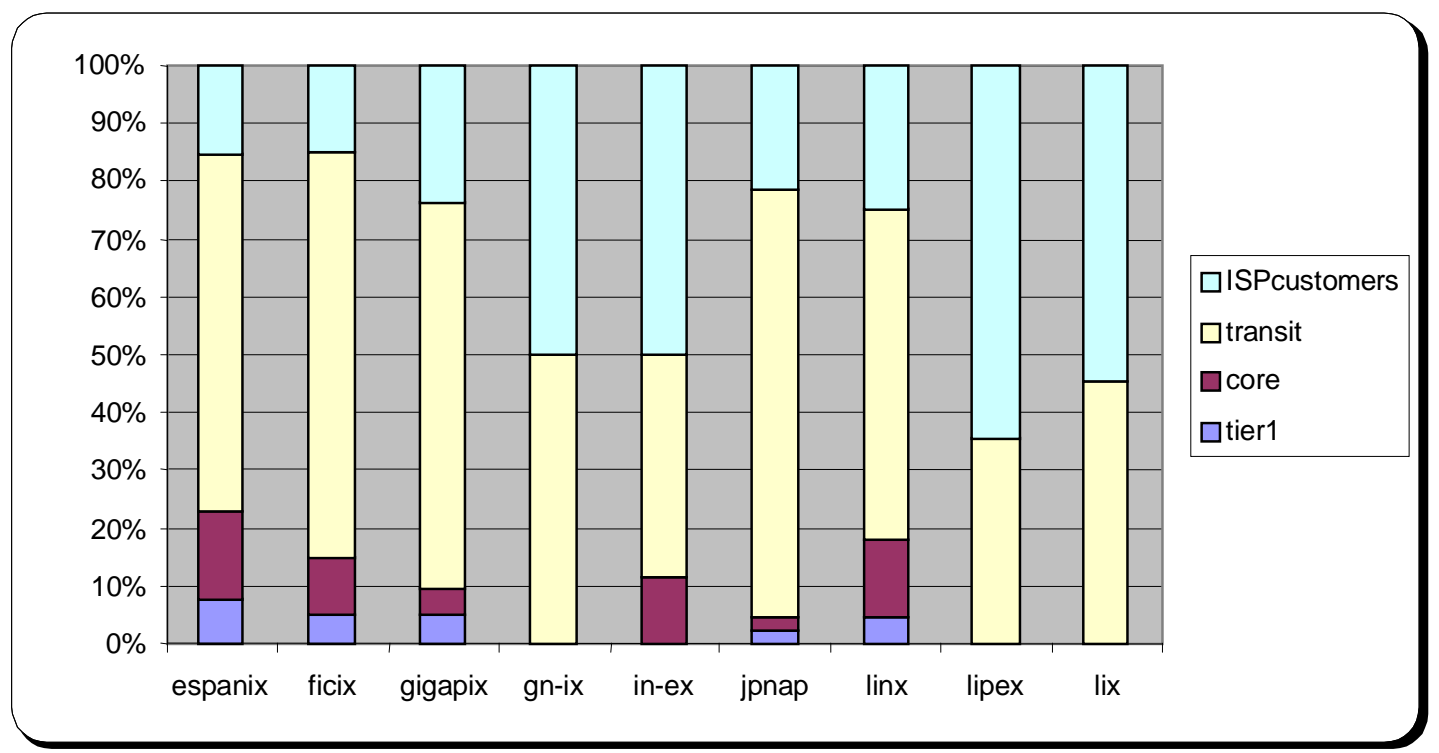


Figure 2.c: IXPs class composition

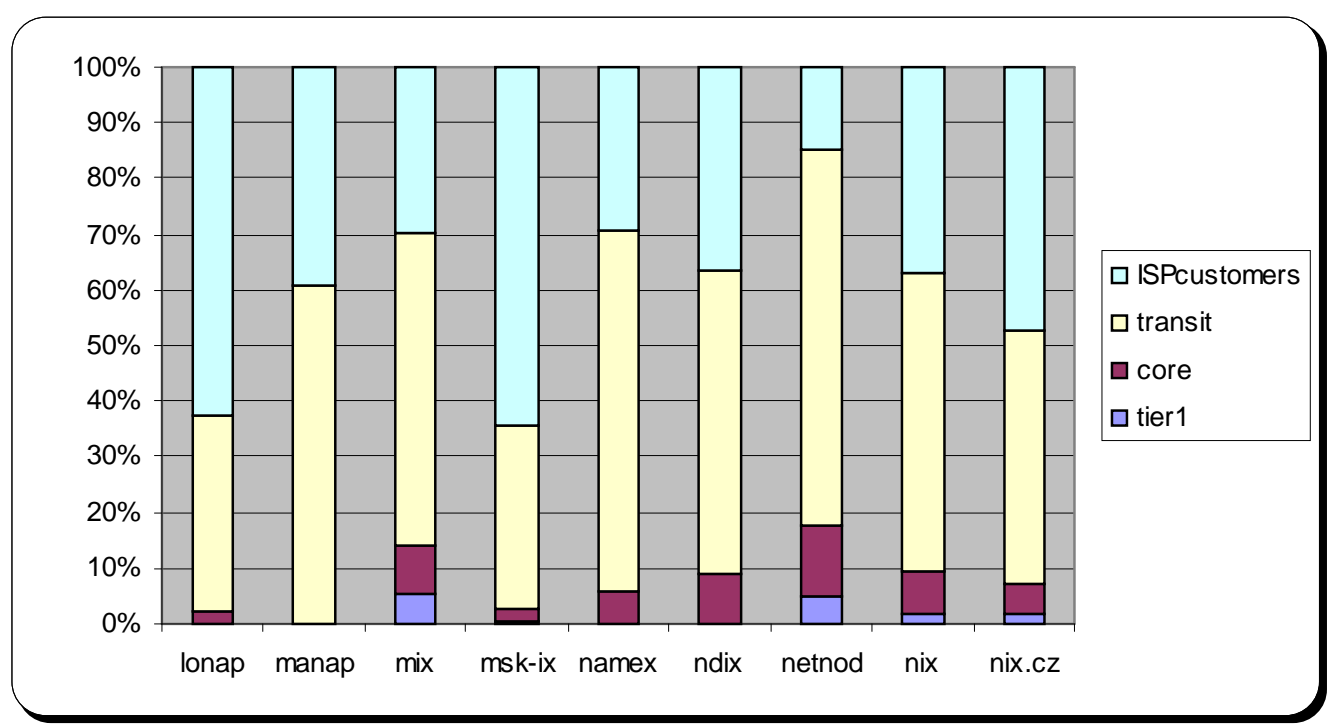

Figure 2.d: IXPs class composition

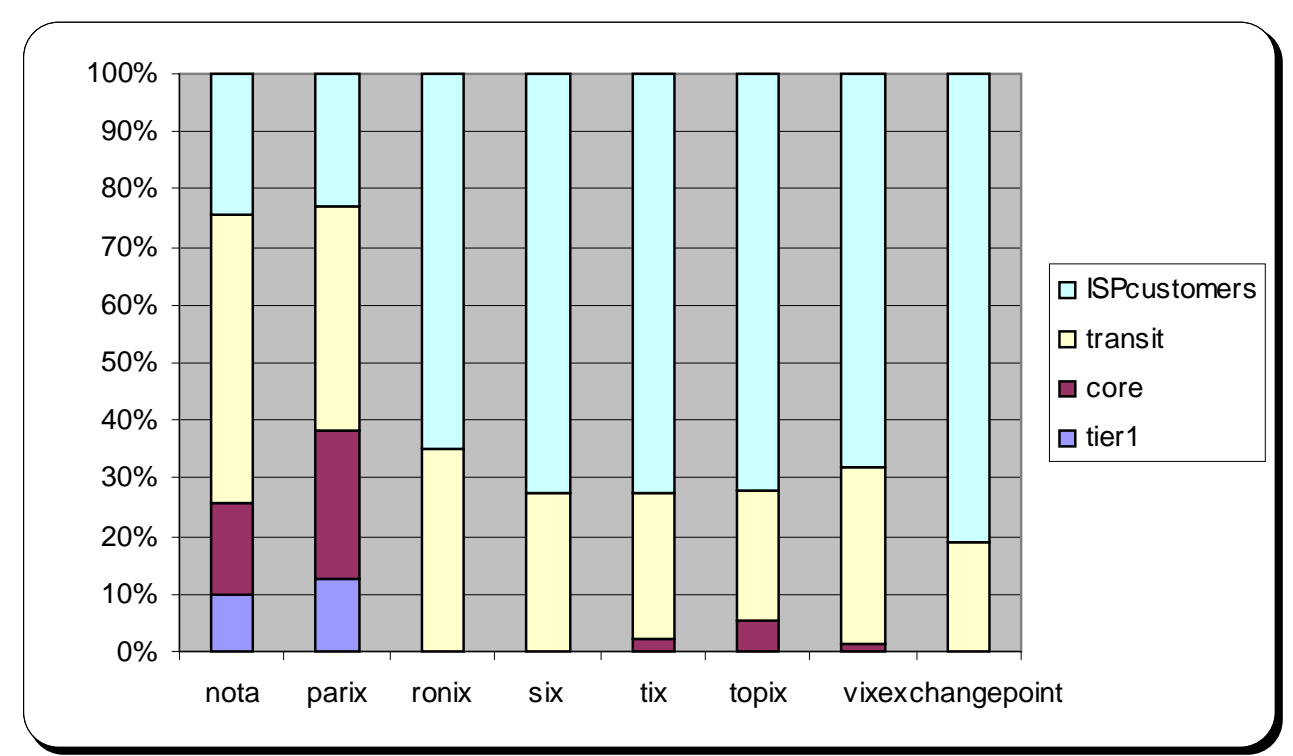


From the Figures $2 \mathrm{a}$, to $2 \mathrm{~d}$, above we notice that less than $50 \%$ of the IXPs (16 out of 35 of the Euro-IX ) have ISPs members belonging to the Tier1 class. This outcome is relevant since it shows that the largest percentage of IXPs is not independently able to forward packets to the entire Internet, i.e. it does not allow direct universal connectivity.

It is also interesting to point out that the IXPs with a small number of members are consistently characterized by a higher percent of ASes belonging from the lowest hierarchies in the Internet (ISP customers). Given the hierarchical structure that governs the Internet, it is presumably sensible to assume that a balanced IXP is characterized by a distribution of ASes over the four classes following a pyramidal structure: the Tier 1 providers should represent the smallest percentage, then a greater share of the members should be constituted by the Core providers, and then the largest share by Transit and ISPS customers providers. Such a balanced structure, however, seems to be a feature of a small set of IXPs. Among the largest IXPs, it seems fairly satisfied, with the only exception of MSK-IX and EXCHANGE POINT. On the other hand, small IXPs seem characterised by consistently different distributions, with few exceptions (BNIX, ESPANIX, CIXP, FICIX, NETNOD).

The bulk of IXPs is low-hierarchy biased, with more than $90 \%$ of members belonging to classes of Transit and ISP customers. The opposite situation characterizes only a few IXPs, where the percentage of providers in the first classes is greater than 20\% (NOTA, PARIX, CIXP, EQUINIX, ESPANIX).

\section{Market Concentration, by vertical and geographical classification.}

Having introduced the vertical market classification algorithm we finally need to consider the problem of drawing the geographic market boundaries before being able to perform our empirical analysis of the Upstream Internet Routing European market. Should we demarcate markets following the national boundaries or is this concept not appropriate for the Internet upstream connections?

In this section we suggest that the most appropriate criterion for geographic demarcation is to identify the IXPs that are independently able to supply universal connectivity, i.e. IXPs having, among their members, ISPs falling into the Tier 1 class. Figure 3 below maps the full geographical distribution of the ISP typologies across IXPs in Europe. The first clear element from this map is that not all locations have Tier 1 class 
providers, being therefore unable to provide independently Universal connectivity, at least within their IXPs memberships.

\section{Figure 3: ISP distribution per typology and IXP location.}

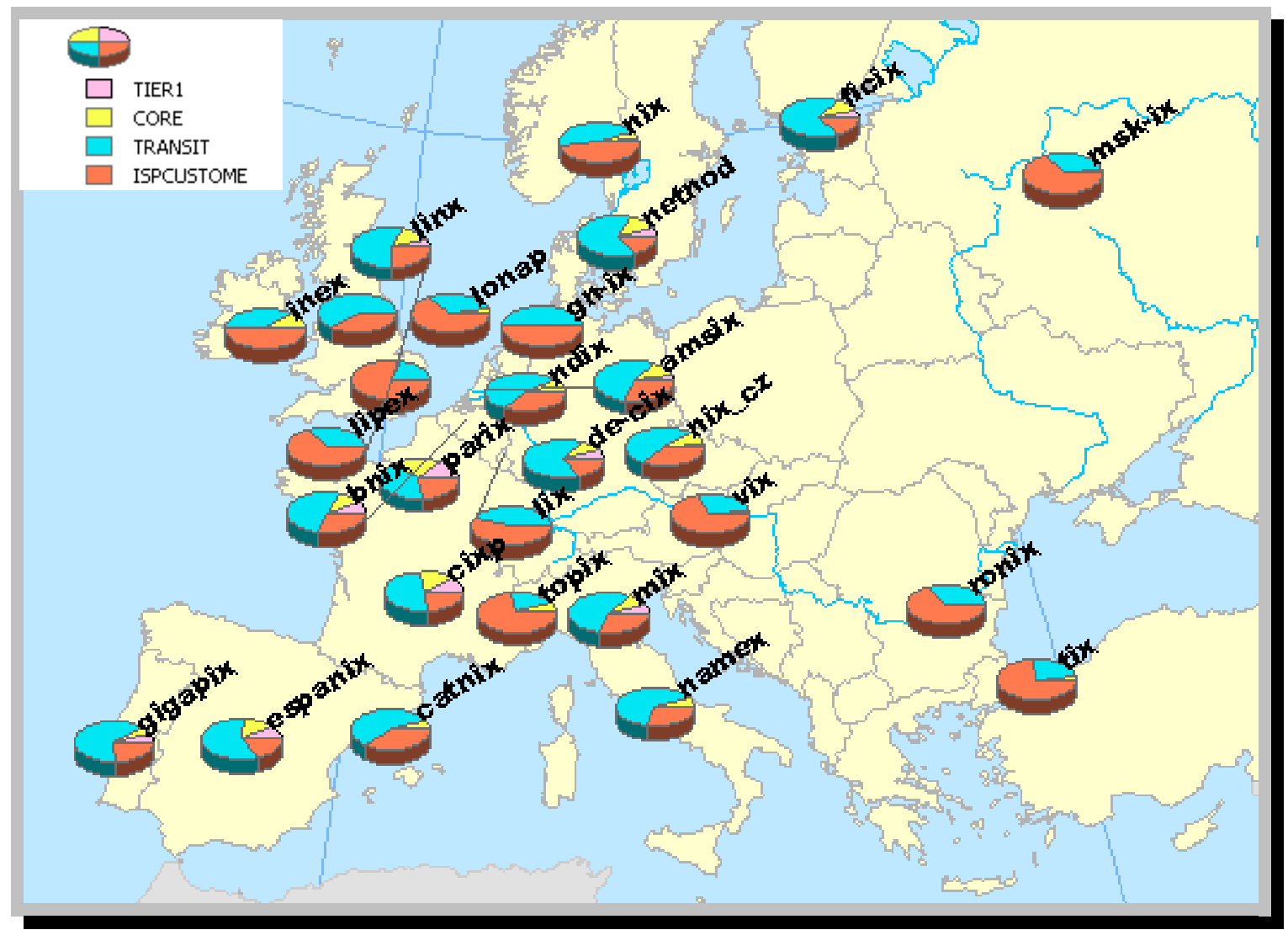

As a result locations related to an IXP unable to provide universal connectivity should not be considered as independent markets.

In the following we focus our attention to a restricted set of geographically independent locations where IXPs have, indeed, the possibility to operate as centres for universal connectivity, without having to connect with another Exchange Point. We consider four of the major IXPs: namely the LINX, DECIX, MIX and AMSIX. Each one of them, having Tier 1 class members, is considered as an independent geographic market allowing direct universal connectivity to its members. For each one of these IXPs we calculate the two specific HHI market concentration indexes discussed in the previous sections, the customer cones and the betweenness one. Moreover, these indexes are also 
decomposed according to five ${ }^{27}$ vertical-hierarchical classes: Tierl (rank 1-12), Inner Core (rank 13-49), Outer Core (rank 50-250), Transit (rank 251-4000), and ISP customers (rank 4001-). As a result we obtain location \& layer specific concentration indexes or, in other words, concentration indexes for the relevant market. The results we obtain, shown in the Tables 3, 4, 5 and 6 below are significantly different from the aggregate ones and we believe they provide a step forward towards the application of the Commission's guidelines in assessing location \& class specific market concentration for the Internet Upstream Routing at European IXPs.

Table 3 below shows the calculations for the LINX IXP.

Table 3: LINX location-class specific Concentration Indexes

\begin{tabular}{lrrr}
\hline Class & \#members & hhi_c_c & hhi_b \\
\hline Tierl (1-12) & 8 & 1253.19 & 1981.51 \\
Inner Core (13-49) & 20 & 416.67 & 616.89 \\
Outer Core (50-250) & 26 & 1037.07 & 1711.95 \\
Transit (251-4000) & 63 & 324.95 & 452.2 \\
ISPcustomers (4001-) & 34 & 303.25 & 471.27 \\
LINX Matrix & 151 (actual & \\
& & 241.18 & 460.797 \\
\hline
\end{tabular}

The first column of Table 3 above, shows the number of IXP's members belonging to each single class, while the second and third columns, provide the two different HHI indexes, the first calculated on the customer cone "market shares" and the second on the betweenness ratios. According to the Merger Guidelines, [U.S. Department of Justice (1997)], some of the market concentration values estimated in Table 3 would immediately fall within the special attention category, in particular we can see that both the first, Tierl, and third, Outer Core (50-250), classes at the LINX, display an HHI concentration index higher than 1000, while class two, Inner Core (13-49), four, Transit (251-4000) and five ISP customers (4001-) appear more competitive.

Moving to AMSIX in the Netherlands, Table 4, below shows that again the first class is the most concentrated, however class three is very concentrated in terms of betweenness.

\footnotetext{
${ }^{27}$ For this application we have subdivided the original Core class introduced above into two classes: Inner Core (composed by ASes ranking between 13 and 49) and Outer Core (composed by ASes ranking between 50 and 250), leaving the other classes unchanged.

${ }^{28}$ ASes with insufficient information in terms of rank or interconnection agreements were deleted.
} 
The difference between HHI based on customer cones and betweenness needs further scrutiny in future research. Surely the betweenness expresses the presence of partly essential facilities, and concentration in it could express the pivotal role of some ISPs that, while not having a particularly large customer base, still represents an almost non-avoidable essential facility in the Upstream Internet Routing.

Table 4: AMS-IX location-class specific Concentration Indexes

\begin{tabular}{lrrr}
\hline Class & \#members & \multicolumn{1}{c}{ hhi_c_c } & \multicolumn{1}{c}{ hhi_b } \\
\hline Tierl (1-12) & 7 & 1432.62877 & 2244.74908 \\
Inner Core (13-49) & 21 & 526.31613 & 746.90882 \\
Outer Core (50-250) & 25 & 840.45206 & 1916.51481 \\
Transit (251-4000) & 77 & 302.661 & 298.13832 \\
ISPcustomers (4001-) & 44 & 285.12583 & 370.29314 \\
AMS-IX Matrix & 174 (actual) & 251.46 & 512 \\
\hline
\end{tabular}

Table 5 below explores concentration at the DECIX.

Table 5: DECIX location-class specific Concentration Indexes

\begin{tabular}{lrrr}
\hline Class & \#members & \multicolumn{1}{c}{ hhi_c_c } & \multicolumn{1}{c}{ hhi_b } \\
\hline Tierl (1-12) & 8 & 1253.18843 & 1866.04104 \\
Inner Core (13-49) & 13 & 769.23147 & 1124.47828 \\
Outer Core (50-250) & 20 & 1078.14838 & 2688.13068 \\
Transit (251-4000) & 80 & 353.57891 & 298.05377 \\
ISPcustomers (4001-) & 21 & 753.55818 & 1130.98521 \\
DECIX Matrix & 142(actual) & 331.97 & 665.02 \\
\hline
\end{tabular}

This Exchange Point shows higher concentration clearly in class one and three according to the customer cone HHI, but also in class one, two, three and five according to the betweenness HHI. Finally, table 6 below describes market concentration at the MIX in Milan. In this IXP we have almost always a very concentrated market structure. Maybe this result is due to the smaller membership characterising each class. 
Table 6: MIX location-class specific Concentration Indexes

\begin{tabular}{lrrr}
\hline Class & \#members & hhi_c_c & \multicolumn{1}{c}{ hhi_b } \\
\hline Tierl (1-12) & 3 & 3333.33 & 6011.1584 \\
Inner Core (13-49) & 5 & 20000 & 3370.93 \\
Outer Core (50-250) & 6 & 2479.1 & 7898 \\
Transit (251-4000) & 26 & 1370.3 & 1119.5 \\
ISPcustomers (4001-) & 17 & 817.8 & 1137.1 \\
MIX Matrix & 57 (actual) & 833.2 & 1896.7 \\
\hline
\end{tabular}

\section{Conclusions}

Concerns about the presence and the effects of market power involving the Internet upstream access are increasing with the fast development of the Internet demand. Antitrust authorities, involved in the analysis of proposed mergers, however lack reliable data, since both traffic flows and interconnection clauses are sealed under confidentiality agreements. In this paper we suggested a possible solution to this problem, centred on the use of innovative metrics to assess concentration in the upstream Internet market. In particular, our approach is based on the retrieval of implicit interconnection policies, the discrimination blueprints expressing the real bargaining power of each ISP, from publicly available Border Getaway Protocol (BGP) data. Indeed, given the confidentiality of explicit peering and interconnection pricing policies for this market, we believe that the only possible way to learn about the economic type of relationship between two undertakings is by examining data contained in the BGP tables.

We focussed on the European upstream Internet market. In order to assess concentration we considered four leading European Internet Exchange Points (IXPs): the London Internet Exchange Point (LINX), The Deutsche Commercial Internet Exchange Point (DECIX), the Amsterdam Internet Exchange Point (AMSIX) and the Milan Internet Exchange Point (MIX).

We followed a two step process: firstly we introduced a price-independent algorithm for defining both the vertical and geographical relevant market boundaries, then we calculated market concentration indexes using two novel metrics. These assess, for each undertaking, both its role as an essential network facility, thorough the measurement of its relative betweenness, and its wholesale market share, via the ranking of its customer cone. 
The results show that some vertical segments of these markets are highly concentrated and would hence fall within the special attention category according to the Merger Guidelines [U.S. Department of Justice (1997)]. The measures of market concentration obtained using our two different metrics tend to move closely together, although with different concentration indexes. This result reinforces the rationale to look at both these dimensions of market concentration. This is expected in the framework of network industries, where a relevant notion of market concentration needs to be captured along more than one single dimension. In our case the two dimensions considered are wholesale customer base, and network centrality.

Finally, market power can be a transient phenomenon, and market concentration may change rapidly in highly innovative sectors, such as the Internet. This clearly implies that the tasks of identifying the relevant market and to assess its concentration need to be re-evaluated regularly. Our proposed indicators can be of particular use for repeated antitrust analysis since they are not based on ad hoc information gathering but on existing algorithms applied to regularly updated databases. As a result we think that no reliable empirical alternative exists to the analysis of the micro-routing decisions, based on the retrieval of BGP policies, for the study of the upstream Internet, routing, market structure, and for its antitrust analysis.

\section{References}

1. Brandes, U. (2001). “A Faster Algorithm for Betweenness Centrality”. Journal of Mathematical Sociology, vol. 25, 163-177.

2. Buccirossi, P., Ferrari, L. and Siciliani, P. (2005) "Competition in the Internet Backbone Market". World Competition 28/2, 235-254.

3. Cave, M. (1999). "Interconnection and the Internet: Competition and Regulation Issues at Local Access and Backbone Levels" OECD, Working Party on Telecommunication and Information Services

4. Claffy, K., Tracie, E. McRobb, M. \&D. (1999), "Internet Tomography”, Nature 7 January 1999. 
5. Cossa, B. (2000) "Economic and policy analysis of mergers among Internet backbone providers" Master of Science in Technology and Policy Thesis, Massachusetts Institute of Technology.

6. Dimitropoulos, X.; Krioukov, D.; Huffaker, B.; Claffy, KC; Riley, G. (2005) "Inferring AS Relationships: Dead End or Lively Beginning?"

7. Gao, L. (2001) “On Inferring Autonomous System Relationships in the Internet”. In IEEE/ACM trans. Networking.

8. Giovannetti, E. Ristuccia, C.A. (2005) "Estimating Market Power in the Internet backbone using the I.P. transit Band-X database," Telecommunications Policy, vol 29 pp 269-284.

9. Huber, B.; Leinen, S.; O’Dell, R.; Wattenhofer, R. (2004) “Inferring AS Relationships Beyond Counting Edges” D-INFK Tech Report Nr. 446.

10. Official Journal Of the European Commission (2000), Regulation (EEC) N 4046/89, Merger Procedure, Bruxelles, European Commission, DGXIII

11. Scheffman, D. T. and Spiller, P. T. (1987). "Geographic Market Definition under the U.S. Department of Justice Merger Guidelines," Journal of Law \& Economics, University of Chicago Press, vol. 30(1), 123-147.

12. Shimbel. A. (1953). Structural parameters of communication networks. Bulletin of Mathematical Biophysics, vol. 15, 501-507.

13. U.S. Department of Justice (1997), The Horizontal Merger Guideline, issued April 2, 1992 and revised April 8, 1997; originally issued as part of US Department of Justice Merger Guidelines, June 141984.

14. U.S. Department of Justice (2000a), Civil Action No. 1:00CV02789 against WorldCom and Intermedia.

15. U.S. Department of Justice (2000b), Civil Action No. against WorldCom and Sprint

16. Werden, G. J. and Froeb, L. M. (1994). "The Effects of Mergers in Differentiated Products Industries: Logit Demand and Merger Policy," Journal of Law, Economics and Organization, Oxford University Press, vol. 10(2), 407-426. 